\title{
Patient Safety Culture of Iganga, Kamuli Mission and Kakira Hospitals of South Eastern Uganda
}

\author{
Article by Balidawa John \\ District Health office Jinja District Local Government, Uganda \\ E-mail: balidawajohn@gmail.com
}

\begin{abstract}
Background: Health care is one of the most important services that every one desires to be of high quality and safety, but in real practice, this is not always the case. In the event of seeking health care, many patients are harmed and some get serious disabilities or even death. System failures and blame for errors committed are the ones that mostly promote occurrence of medical errors hence resulting into unsafe healthcare. Blame prevents health care providers from reporting errors for future prevention of re occurrence.

Patient safety, which is a component of quality healthcare, is defined as the absence of avoidable harm to patients during the process of health care, (Carmen A. n.d.). To achieve 100\% absence of avoidable harm to patients is hard to realise as 'to err is human' but efforts to avoid harm are of great importance. In developed countries, thousands of patients are reported to suffer serious harm and death in the event of receiving health care services. In 1999, Institute of Medicine in the USA estimated 44,000 to 98,000 preventable deaths annually due to medical errors in USA hospitals, (IOM 1999). In Uganda`s healthcare system, many patients are harmed and many even die during the event of seeking for healthcare but due to poor reporting systems there is limited information on the magnitude of preventable harmful medical errors.

The study to determine patient safety culture of Iganga, Kakira and Kamuli Mission in south eastern Uganda, had five objectives of; assessing the knowledge of health workers on patient safety, the role of the hospital management in promotion of patient safety culture, determine patient safety culture practices, point prevalence of common inpatient safety incidents, and causal pathways.

Methodology: The study was an observational, descriptive and cross-sectional survey. A sample of 144 health workers were interviewed using a questionnaire, three focus group discussions with hospital managers were conducted, and 169 inpatient records were reviewed. Data was entered in SPSS 16. 0 software then into Excel for further analysis and later presented in graphs.

Results: The study revealed that the knowledge of health workers on patient safety in the study hospitals was just above average at 55.8\%. Under and over dosing of patients, dispensing of wrong drugs and poor infection control being the most common medical errors known, where as Uganda clinical guideline was found to be the most known guideline for patient safety. The study also showed that, the majority of health workers would not report errors due to ignorance and fear of blame. The study showed existence of team work and channels for communication of patient safety issues between management and staff. However, management role in promotion of patient safety is still demanding as there was limited availability of policies and guidelines, lack of patient safety incident record books and patient safety committees. There existed fairly good patient safety practices as communication, hand washing, dispensing and team work were all above average. However it was found out that poor reporting, fear of blame, under staffing and inadequate waste disposal practices did exist. Incidents of failure to monitor vital signs and delayed investigations were more prevalent in over $70 \%$ of all records studied. The majority of the surgical operations were done without pre-operative investigations and had inadequate post operative notes increasing the chances of harm to surgical patients. Causal pathways for medical incidents were mainly lack of guidelines and policies, poor monitoring of adherence to guidelines, limited skills and training of staff, understaffing, heavy
\end{abstract}


workloads, lack of logistics and faultiness of equipments and lack of reporting procedures for patient safety.

Conclusion: In conclusion, the level of knowledge on patient safety culture is just average as awareness is still limited. There is a significant lack and limited availability of policies and guidelines, incident record books and committees that promote patient safety. The bad practices of fear to report incidents, under-staffing and fear of blame for errors committed need improvement. Failure to monitor vital signs, difficulty to access high skilled staff especially the medical officers and inadequate post operative notes also need serious attention as they are part of the causal pathways for patient safety incidents in the study hospitals. Provision of policies and guidelines, training of health worker, management leadership and effective communication and monitoring of patient safety in the study hospitals are highly recommended.

\section{Introduction}

Patient safety is one of the dimensions of quality of care and it is defined as the absence of avoidable harm to patients during the process of health care (Carmen, n.d.). The international classification for patient safety defines patient safety as "The reduction of risk of unnecessary harm associated with health care to an acceptable minimum" (WHO, 2009a p133).

The Department of Health Expert Group in June 2000 estimated that over 850,000 incidents used to harm hospitalized patients in the United Kingdom alone each year, (Department of Health Expert Group, 2000). In 2004, the Canadian Adverse Events Study found that adverse events occurred in more than 7\% of hospital admissions, (Ross B et al, 2004). Such reports have led the World Health Organization to estimate that one in ten persons receiving health care suffer a preventable harm (WHO, n.d.a).

Patient safety involves all aspects related to provision of harmless healthcare to patients such as avoidance of medical errors, incident reporting, adherence to standard guidelines, policies and procedures for patient safety, adequate training of staff, effective communication structures, and patient safety monitoring and evaluation.

Quality assurance in the health sector in Uganda started recently in 1994 as the quality assurance program, which was created to support health service delivery in a decentralized system. The Uganda Ministry of Health supported the Yellow Star Program as a major quality management intervention, which focused on minimum service standards for a range of Primary Health Care services. However, there is a relatively little experience in measuring patient safety culture and improvement in the Ugandan healthcare system.

\section{Justification}

World Health Organization estimates that one in ten hospitalized patients are harmed in the process of provision of healthcare. Uganda's hospitals are not exceptional given the standard of her healthcare system. Organization systems that promote patient safety have improved over years in the developed world, but patient safety measures have remained poor in the developing countries, including Uganda. It is hard to give concrete figures to what extent poor patient safety has affected patients in developing countries simply because of limited documented data and information accessed. However, in Europe, a number of studies carried out confirmed that patient safety was the major concern for that region by 2007, according to the European Union Patient Safety Working Group (EU-PSWG, 2007).

There is limited information on the level of health workers ' knowledge on patient safety, the role manager's play in the promotion of patient safety, patient safety practices, and the prevalence of inpatient safety incidents and their causal pathways in the study hospitals of Kakira, Iganga, and Kamuli Mission. Without this information, patient safety improvement would be hard to be realised and many patients would continue suffering harm and deaths in the process of seeking health care.

The study was meant to build on the knowledge of the researcher, the health workers, Ministry of Health and other stake holders on the level of patient safety in general hospitals so has to seek improvement. This information if used by the hospital managers and health workers will help the 
studied hospitals to plan and improve on their patient safety practices. The Ministry of Health can now benefit from the research findings, if used, by understanding the patient safety culture in the public and private general hospitals of eastern Uganda, to influence policy and guidelines on patient safety. Other stake holders like WHO can also have additional understanding of patient culture in the Ugandan hospitals.

\section{Background of the study area}

The study established the patent safety culture in general hospitals of Iganga, Kakira and Kamuli mission, that were conveniently selected. Iganga Hospital is a public general hospital, Kakira Hospital is a Private For Profit and Kamuli Mission Hospital is a Private Not For Profit hospital.

Study Goal:

To assess the patient safety culture of public and private general hospitals of south eastern Uganda to generate information that if used can contribute to patient safety improvement in Uganda.

\section{Objectives of the study}

To assess the knowledge of health workers on patient safety in the study hospitals.

To assess the role of the hospital management in promotion of patient safety culture in the study hospitals.

To assess the patient safety culture practices in the study hospitals.

To determine the point prevalence of common inpatient safety incidents in the study hospitals

To determine the causal pathways for the common inpatient safety incidents in the study hospitals.

\section{Study type, population, unit and sample size}

The study was an observational, descriptive and cross sectional survey, both qualitative and quantitative in nature. The study population was three general hospitals of South Eastern Uganda of Iganga, Kamuli mission and Kakira. The study units were the health workers in the different departmental units of a hospital and inpatients records. The sample size of the health workers was 143 determined by use of the hyper geometric method for a known population. A census of inpatient records for patients admitted at least 24 hours to the time of survey was conducted to determine the prevalence of patient safety incidents.

\section{Data analysis and presentation methods}

Data was collected, collated and entered into SPSS 16.0 software to get tables of frequencies and percentages. Data was transferred into excel for further calculations and lastly to word from where narrative interpretations were made.

\section{Ethical considerations}

Consent was by respondents signing the questionnaire before completing it. The participants/respondents were also reassured of confidentiality to the information that was obtained from them.

\section{Results and analysis}

\section{Socio-demographic characteristics of respondents}

The percentage of health workers trained in patient safety was that; Iganga hospital had $54.5 \%$ ( $\mathrm{n}=44)$, Kamuli mission had 52.4\% ( $\mathrm{n}=42)$ and Kakira hospitals had 62.7\% ( $\mathrm{n}=51)$.

\section{Distribution of inpatient files reviewed for patient safety incidents per ward. $(n=169)$}

Iganga hospital had the highest number of files studied of 58.6\%, followed by Kamuli Mission with $25.4 \%$ and lastly Kakira hospitals with $16 \%$. This was because Iganga hospital has more workload of patients than Kamuli and Kakira. The more, the patients that are handled, the more the pressure build 
up on the hospital resources and hence the likely hood of occurrence of medical mistakes. However the staffing levels of Iganga also observed to be higher than for other hospitals. All the wards were represented by the inpatient files at the time of survey with children`s ward having more files reviewed than other wards. This means that more children get admitted than adults in the study hospitals except for Kakira hospital that had more of males admitted due to traumas, given the nature of work of working in a factory.

\section{Knowledge of health workers and hospital managers on patient safety}

Health workers able to explain what patient safety is; $32.8 \%$ of health workers interviewed explained well what patient safety is, with Kamuli mission hospital having slightly a higher percentage of $35.1 \%$, than Kakira and Iganga hospitals with $34.8 \%$, and $28.2 \%$ respectively. Kakira hospital that had the highest percentage (32.8\%) of staff trained in patient safety than the other hospital, on the other hand had a few health workers aware of what patient safety is all about.

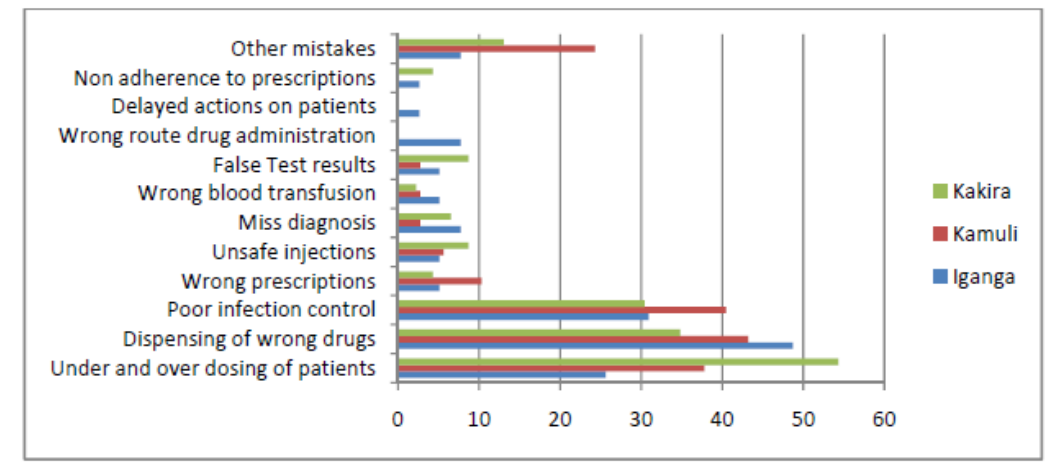

Figure 1. Common medical mistakes known by health workers in percentages. $n=122$

It was noted that $76 \%$ of the respondents from all the study hospitals could mentioned at least two common medical errors known to them. Under/over dosing of patients, dispensing of wrong drugs and poor infection control were the most known medical errors in all the study hospitals as noted in figure 1.

\section{Measures in place for prevention of medical errors reported. $(n=122)$}

Generally, only $55.3 \%$ of the respondents could mention at least two measures in place to prevent medical errors, and a big number of health workers were not aware of the measures in place.

Iganga hospital had the highest percentage (68\%) of respondents mentioning at least two measures in place for prevention of medical errors, followed by Kamuli (52.7\%) and lastly Kakira (46.7\%). Iganga hospital with the least number of staff who could explain well what patient safety is all about, had the highest percentage of staff mentioning at least two measures for prevention of medical errors.

Good patient care practices and observation of infection control were the most reported measures used to prevent medical errors in the study hospitals. Use of CMEs to prevent medical error was reported by less than $20 \%$ of the respondents, yet the researcher thought that it could be mentioned by more staff.

Use of diagnostic service, training and use of skilled staff were least mentioned as measures used to ensure patient safety in the study hospitals. This means that health workers in the study hospitals are not aware that these are measures that also contribute to promotion of good patient safety in health care, so the need for sensitization of staff on these measures. 


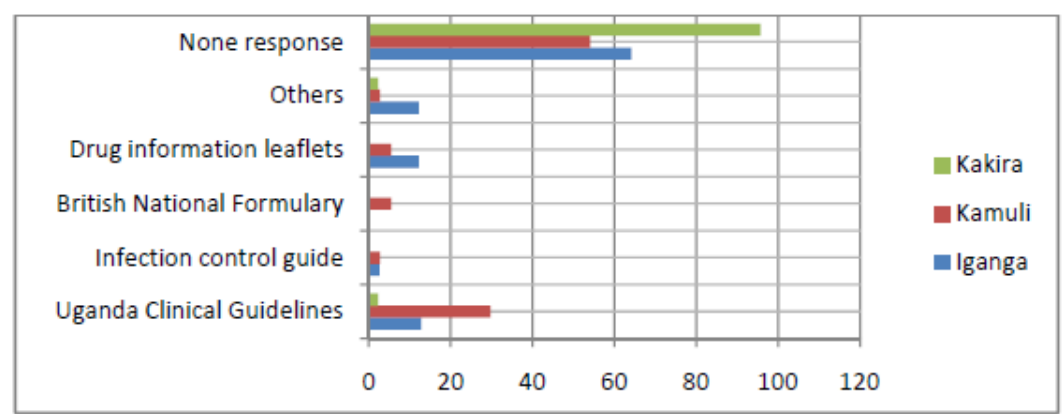

Figure 2. Known policies, documents or guidelines used to ensure patient safety in percentages. $n=122$

Generally, there were few respondents who could mention at least two policies or guidelines used to promote patient safety. Only 18\%, 23\% and 2\% of Iganga, Kamuli and Kakira hospital health workers respectively would mention at least two policies used. Uganda clinical guideline was the most known document used, compared to infection control guidelines, British National Formulary and drug information leaflets. Kamuli hospital that had more than two copies of the Uganda clinical guidelines in use at the time of survey and more health workers were aware of the policies and guidelines used for patient safety. Kakira hospital that had none of the studied policies/guidelines had the least percentage (2\%) of her health workers aware of such documents. This means that at Kakira hospital there is limited use of policies and guidelines that promote patient safety.

Report practices for medical errors; It was observed that 57.8\% of health workers would report all medical errors committed at the study hospitals. In Iganga hospital, $60.9 \%$ of its health workers would report all medical errors committed as compared to $54.7 \%$ and $58 \%$ of Kamuli and Kakira respectively. This means that a big percentage of errors committed in the study hospitals would go unreported. It is also noted that medical errors with severe harm would be reported more than the ones with no or less severe harm. This can be attributed to the fact that errors with severe harm require involving other high level skilled staff to manage.

\section{Reasons for reporting medical errors}

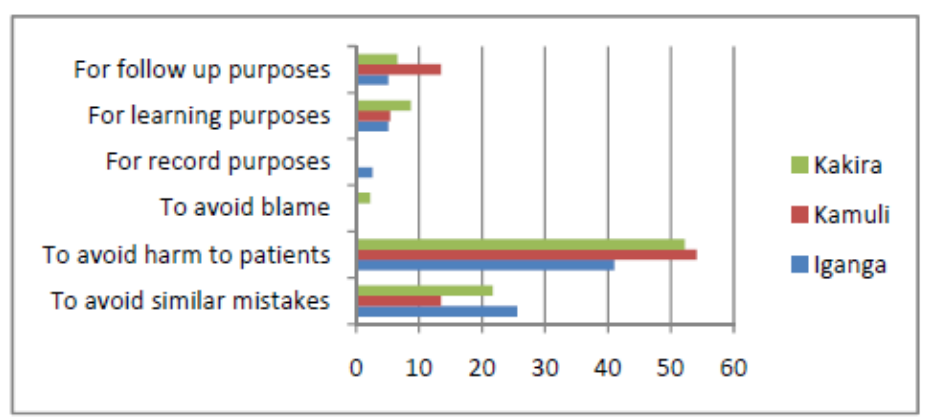

Figure 3 Percentages of reasons for reporting medical errors. $n=122$

More than a half of the respondents $(\mathrm{n}=122)$ would report errors to avoid harm to patients and less than $25 \%$ of respondents would report to avoid similar incidents in future. $79.4 \%(n=44), 86.5 \%$ $(\mathrm{n}=42)$ and $91 \%(\mathrm{n}=51)$ of respondents of Iganga, Kamuli and Kakira hospitals respectively gave at least a correct reason as to why they would report errors. The rest would not report medical errors because of not knowing the importance of reporting and fear of blame.

Conclusively, the level of knowledge on patient safety at the study hospitals was just above average as Iganga hospital scored 55.3\% ( $\mathrm{n}=44)$, Kamuli mission and Kakira hospitals scored $57.8 \%$ $(\mathrm{n}=42)$ and $54.1 \%(\mathrm{~N}=55)$ respectively. 


\section{Managers' role in promotion of patient safety culture}

The manager`s role in promotion of patient safety was studied using six indicators as described in narrative form here under. It was assessed using both observational tools and likert scale grading of the level of agreement to the statements in the questionnaire.

\section{Availability of documents/policies/guidelines that promote patient safety culture}

The study observed that Iganga and Kamuli hospitals had at least a copy of the Uganda clinical guidelines 2010. The fact that only one copy of the Uganda clinical guidelines was available at OPD of Iganga hospital at the time of survey, it means that accessibility to this copy by the rest of the clinicians in their common consultation room was limited. It is only Kamuli Mission hospital that had an Injection Safety Policy in place so it can be taken to have better injection safety practices than Iganga and Kakira hospitals.

Kamuli and Kakira hospitals had patient vital observation charts to promote observation of patient vital signs; whereas Iganga uses un formatted exercise books for recording such information. It was noted that despite the availability of these charts, they were not being used by Kakira hospital and to some extent they were being used by Kamuli Mission hospital. The use of these charts to monitor vital signs in Kamuli Mission hospital was attributed to the practice of the hospital and availability of student nurses and midwives. It was surprising that despite the availability of student nurses from Busoga University at Iganga hospital, there was no patient observation charts for students to practice from. This may result in Busoga university producing nursed with limited skills in observation of vital signs. The limited use of the observation charts in Kamuli Mission hospital was due to break down of equipments used to monitor such signs. Kakira hospital management had not enforced the use of the available patient observation charts as health workers were not interested in completing them.

All the hospitals lacked a used parto-graph, and this means that women who had their labour and delivery in the study hospitals were not being monitored as recommended by the Uganda Ministry of Health. This practice could be resulting into avoidable maternal and infant deaths at these study hospitals. At all the study hospital pharmacies, there was no patient information charts on medicines displayed. This means that patient at the study hospitals cannot easily identify possible problems that they could face in the process of medication and may use drugs irrationally. It was a new term at the study hospitals when I asked about WHO Safe Surgery Saves Lives check-list to check if it was available. All the theatre in charges acknowledged not having heard of such a document in their life time of practice. This means that the chances of misidentification of patients for surgery are high in the study hospitals, so a wrong patient or site could get operated. It can be concluded that the study hospitals are to some extent unsafe for surgical patients.

Lastly, none of the hospitals had a patient incident record book to track occurrence of incidents in the hospital. This means that there is limited means of monitoring patient safety incidents and learning from them.

\section{Monitoring staff adherence to policies and guidelines by management}

The hospital managers of the study hospitals reported monitoring adherence to patient safety guidelines through, support supervision, conduction of patient satisfaction surveys and meetings. However these means of monitoring were not verified to determine to what extent they are used by the hospital management. None of the hospitals reported training and building skills of staff in use of the policies and guidelines.

\section{Communication of patient safety issues in the hospital}

It was reported that communication in the hospital on patient safety was mainly through continuing medical education, internal memos, leaflets, meetings, and supervision. This was not also verified to determine to what extent managers communicated to staff about patient safety. Communication of staff to management was reported to be through direct reporting to senior staff, meetings, suggestion 
box, supervisors and during supervisions. None of the study hospitals uses a patient safety incident record book or a patient safety committee because the two channels of communication were not in place in the study hospitals.

Blame free culture by hospital managers; Management blame for error committed prevents reporting of errors and hence learning from errors is affected. All the managers reported that they support their staff to report errors. None of the managers reported blaming a staff and taking a disciplinary action against such a staff for reporting an incident. All hospital managers reported that they apply their own rules and procedures when an incident is reported instead of reporting to other authorities that could punish for the incident.

Availability of patient safety monitoring team or process; Monitoring patient safety improvement is another indicator for commitment to promotion of a good patient safety culture by management. Management mostly reported continuing medical education and internal supervision on patient safety as the commonly used methods for monitoring patient safety in the study hospitals. None of the managers reported having a patient safety committee or a patient safety incident record book in place. Lack of these two measures promotes a poor learning culture from mistakes committed and management may not realise to what extent they could be contributing to unsafe health care in their hospitals.

Health worker's responses on team building by management of the hospital; The study hospitals had good team building practices, however, their supervisors encourage working faster due to work pressure increasing the chances of medical errors.

\section{Patient safety practices practiced in the study hospitals}

Learning from reporting and team working: The study hospital managers reported learning from incidents reported although there was no incident record book in place to verify this practice. The study further noted that the staff of the study hospitals work as a team, they support one another and this reduces on the chances of mistakes that could be committed if there is no team work.

The practice of reporting incidents; The study revealed that the study hospital staff fear being blamed for errors committed although managers reported supporting staff to report errors. The fact that Kamuli and Kakira hospitals are private, with different employment terms from the government hospitals like Iganga, it was surprising that the difference in the practice of reporting errors is small.

Staffing and working conditions; In all hospitals, staffing and working conditions scored below 13 out of 20. Kakira reported having better staffing and working conditions with a score of 12.6, than Iganga with 9.3 and Kamuli with 8.5. This can be attributed to the number of patients managed at each of the hospitals, as Kakira had the lowest number of inpatients (27), compared to Iganga (99) and Kamuli (43) at the time of survey. Limited staffing in a hospital increases the pressure due workload to the few staff. This can lead to stress, de-motivation and limited use of required resources as the aim is to work and finish up all the patients.

Perception of staff on patient safety; All hospitals scored slightly above 12 out of 20 on perception of staff on patient safety, with Kakira hospital having staff with a good perception, than Kamuli and Iganga hospital respectively.

Hand washing practices; Iganga hospital scored slightly just above 9 out of 20 on hand washing practices. This means that the hand washing practices at this hospital need attention. Kamuli and Kakira hospital scored above average. Kakira hospital had better hand washing practices than the other hospitals and this could be contributed to availability of good hand washing facilities. Poor hand washing practices promote cross infections in the hospital and patients end up with health care acquired infections that can increase on bed occupancy period and hospital costs.

Waste Disposal Practices; On dispose of sharps, all the study hospitals scored above 82\%. It was observed that Kamuli hospital had all the required waste management bins, were as Iganga and Kakira hospitals had none. This means that spread of infections from medical waste was limited at Kamuli hospital, compared to Iganga and Kakira hospital. 
Infection Control; Availability of each of the buckets for JIK, soapy water and plain water was noted to be good at Kakira and Iganga and poor at Kamuli Mission hospitals. This can mean that, although Kamuli hospital minimises spread of infections through good waste disposal, it also spreads other blood born infections from patient to patient and also from patient to health worker.

Dispensing Practices; Observation of dispensing time was averagely between 31-60 seconds per patient in each of the hospitals, yet the good dispensing time would be at least more than 60 second per patient. This means that patients are given limited information about the medicines they are given, a practice that increases chances of irrational use of drugs which is a medical error.

\section{Prevalence of medical incidents}

The point prevalence of inpatient safety incidents based on the patient records was found to be $88.7 \%$. This means that almost all the inpatients in the study hospitals at the time of study were at a high risk of suffering from harm due to health care.

Point prevalence of inpatient safety incidents based on patient records $\mathrm{N}=169$

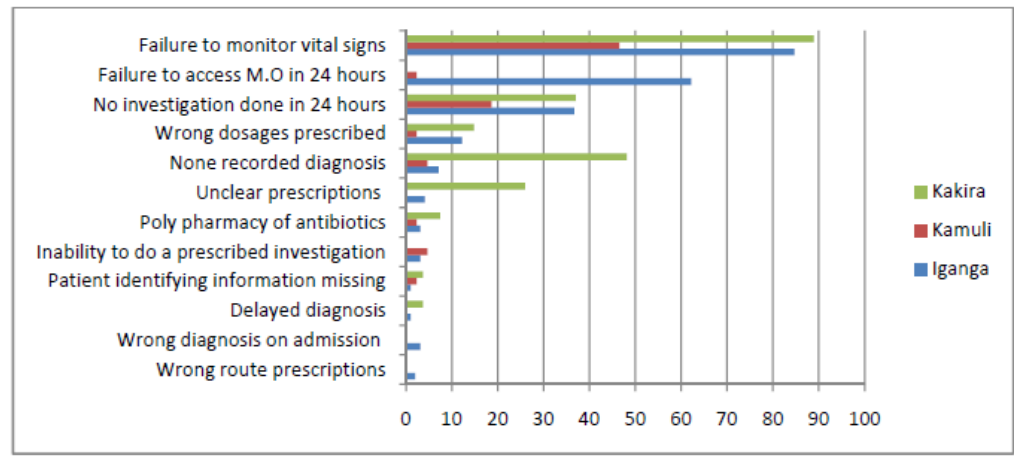

Figure 4. Showing prevalence of patient safety incidents in percentages

The commonly identified incidents were; failure to monitor vital signs of patients and conducting no investigations in 24 hours of admission in over $70 \%$ of inpatient records reviewed. Over $80 \%$ of inpatients of Iganga and Kakira hospitals had their vital signs not monitored, where as only $46.5 \%$ of inpatient of Kamuli missed their vital signs being monitored. It was also found out that $62.2 \%$ of inpatients of Iganga hospital had no access a medical officer in 24 hours of admission as compared to Kamuli (2.3\%) and Kakira hospitals (0.0\%). Kamuli hospital always adhered to the treatment guidelines compared to Kakira and Iganga hospital. Kakira hospital had $25.9 \%$ of its inpatient files with unclear prescriptions mainly due to bad hand writing, compared to $4.1 \%$ and $0.0 \%$ for Iganga and Kamuli hospitals respectively. Kakira hospital had the capacity to investigate most of the requests on the inpatients but Iganga and Kamuli did not, mainly due to logistical problems.

Frequency of Surgical Incidents; It was observed that $62 \%(n=21)$ of files for patients who had surgical operations had no pre-operative laboratory investigations, while $85.7 \%$ of them had inadequate post operative notes and instructions. Kamuli does the basic pre operative investigation with some operations having adequate post operative notes and instructions.

Labour and delivery incidents; Labour and delivery incidents were not common in all hospitals but the fact that parto-graphs were not being used in all the hospitals, it is likely that mothers in labour are suffering from some harm due to poor monitoring of labour. Kamuli Hospital had a big number of babies born with low APGAR score as they reported receiving mothers with babies who were already asphyxiated.

\section{Causal pathways for medical incidents}

Medical Incidents; In Iganga hospital, poly-pharmacy of antibiotic was attributed to multiple coinfections that patients present with, where as in Kakira hospital it was attributed to limited skills of health workers, multiple patient complaints and misdiagnosis. Kamuli reported no such occurrence as 
prescriptions are based on policy guidelines. Unclear prescriptions, were attributed to poor hand writing of prescribers, heavy workload, limited skills of prescribers, prescribers being in a hurry most of the time and differences in origin of training like Kakira hospital that employs Indian trained doctors.

Surgical Incidents: It was also noted that pre operative investigations are not done due to limited logistics, staffing and limited skills. The purpose of doing routine pre operative investigation was not known given that there are no guidelines to support this. The good practice reported by Kamuli hospital was that H.B, blood grouping and cross matching is routine to all admitted patients.

On adequacy of operation notes, it was reported that poor attitude of surgeons towards writing operation notes is one of the causes of writing inadequate post operative notes. It was noted at Iganga hospital were the heavy workload does not allow writing adequate notes. At Kamuli hospital, this was not a common incident as most of their surgeons wrote adequate notes after operation.

Wrong site surgeries were not common in the study hospitals but this does not mean that they do not occur given the fact that none of the hospitals had the checklist for safe surgery.

As for identification of patients for operation, all hospitals reported limited chances of wrong identification of patients for operation as it was reported that surgeons themselves book patients for operation. However, they all accepted that they lacked a checklist to ensure $100 \%$ patient identification for operation.

Preventive Incidents; Missing some basic inpatient identification information was reported to be a common incident. Iganga hospital lacks a format and pre-designed charts to ensure that all information is recorded. Kakira and Kamuli had pre-designed charts and enabled them to fully identify their patients. Too much workload, negligence, understaffing and lack of logistics like blood pressure machines were given as the reasons for failure to observe vital signs of patients on a regular basis. Low APGAR score were being experienced simply because some mother in labour fear to be operated and so they would report late to hospital and also they would take time to accept operations. Lack of logistics would at times cause delay to carry out operations and hence it led to asphyxiated babies delivered. Kamuli hospital reported that low APGAR scores are not expected as there is close monitoring of mothers in labour for any foetal distress so as to take action in time.

Diagnostic Incidents; Due to limited skills and lack of capacity to do investigations for diagnosis, it was reported that it is possible to have a wrong diagnosis. Faulty equipment, limited staffing and poor attitude towards some tests like sputum examination were leading to delayed diagnosis. Under staffing of medical officers, limited their accessibility to patients in Iganga hospital, but it is a routine for medical officers to manage all inpatients in Kakira and Kamuli hospitals.

Investigative Incidents; It was reported that it is not common to have an inpatient to spend more than 24 hours without investigations. However, it can happen where there is limited staffing and lack of logistics to use. Failure to do basic investigations was mainly attributed to lack of basic equipment or due to mechanical problems. Kamuli hospital had a faulty X-ray machine whereas Kakira had no ultra sound machine.

\section{Conclusion}

This study, aimed at assessing patient safety culture in general hospital of South Eastern Uganda, was an observational, cross sectional and qualitative study with 144 respondents and 169 inpatient records reviewed. The study that looked at several aspects of patient safety, observed that the level of knowledge of health workers on patient safety was limited. There is therefore need for training of health workers in prevention of medical error and adherence to the principles and guidelines that promote patient safety. Manager's role in patient safety is crucial especially in enforcing and maintenance of patient safety measures, so the need for managerial leadership in patient safety. There was generally limited availability of policies and guidelines that promote patient safety in the study hospitals and hospital managers are encouraged to develop their own in addition to those developed by government in order to promote patient safety. The prevalence of patient safety incidents of $88.7 \%$ 
is high as incidents of failure to monitor vital signs of patients, conducting no or untimely investigations on patients and difficulty to access high skilled staff were found to be more prevalent. These are preventable with minimal resources required and so manager need to take action.

\section{Recommendations}

The Uganda Ministry of health needs to design training programs for the health workers on patient safety

There is need for leadership in patient safety by the managers to ensure provision and monitoring of patient safety guidelines.

Managers also need to introduce error reporting and evaluation system like introduction of patient safety committees and incident reporting tools.

\section{Acknowledgements}

The following individuals have been acknowledge for their support in the implementation and reviews of the paper; Dr. Dyogo Peter, District Health Officer Jinja and Mutiibwa Tonny health educator Buwenge General Hospital

\section{References}

[1] AHRQ (2007). Closing the Quality Gap: A Critical Analysis of Quality Improvement Strategies. Volume 7, Number 9. www.ahrq.gov. Stanford University-UCSF Evidence-based Practice Center, Stanford, CA.

[2] BATEGANYA MOSES, AMY HAGOPIAN, PAULA TAVROW, SAMUEL LUBOGA AND SCOTT BARNHART 2009. Incentives and barriers to implementing national hospital standards in Uganda. International Journal for Quality in Health Care 2009; Volume 21, Number 6: pp. 421 -426 10.

[3] CARMEN AUDERA. n.d. WHO Patient Safety Programme-Meeting the challenges Faced by Emerging Countries in the Provision of Quality Primary Health Care Cape Town. WHO.

[4] CARMEN AUDERA-LOPEZ 2011. WHO patient safety programme 3rd annual conference on safety, standards and customer services lagos 13th and 14th october 2011. WHO.

[5] DEPARTMENT OF HEALTH EXPERT GROUP 2000. "An organisation with a memory". Department of Health, United Kingdom.

[6] DONABEDIAN A. 2005. Evaluating the Quality of Medical Care. The Milbank Quarterly, Vol. 83, No. 4. Milbank Memorial Fund. Published by Blackwell Publishing

[7] EU-PSWG 2007. Recommendations on patient safety by patient safety working group. European commission health and consumer protect general. Accessed on http://ec.europa.eu/health/archive/ph_systems/docs/ev_20080617_rd01_en.pdf on 8/5/2012.

[8] IAPO (2008a), Uganda Health News: IAPO Launches Patient Safety Toolkit. Kampala, Uganda. Accessed from http://www.ugpulse.com/uganda-news/health/iapo-launches-patient-safetytoolkit/7054.aspx 24/4/2010

[9] IAPO (2008b). Addressing global safety issues. An advocacy toolkit for patients`organization

[10] INSTITUTE OF MEDICINE (IOM). 2000. "To Err Is Human: Building a Safer Health System." In L.T. Kohn, J.M. Corrigan and M.S. Donaldson, eds. Washington, DC: National Academy Press.

[11] LUWEDDE M 2011. Uganda Catholic Medical Bureau Bulletin Jan - Dec 2011. Page 13-15

[12] MOH (2009), Uganda National Health Policy II 2011-2020

[13] MOH (2011), Health Sector Quality Improvement Framework and Strategic Plan 2010/11 - 2014/15.

[14] MOH (2005), Injection safety and appropriate health care waste management. MMIS.

[15] ROSS BAKER, PETER G. NORTON, ET AL. (2004). "The Canadian Adverse Events Study: the incidence of adverse events among hospital patients in Canada". Canadian Medical Association Journal 170.

[16] WHO (2004), World Alliance for Patient Safety, Forward programme 2005. Accessed on http://www.who.int/patientsafety/en/brochure_final.pdf on 8/3/2012.

[17] WHO (2008a), Better knowledge for safe care, Global Priorities for Research in Patient Safety (first edition) The Research Priority Setting Working Group.

[18] WHO (2008b), World Alliance for Patient Safety WHO Guidelines for Safe Surgery. 
[19] WHO (2009a pp106-147), Conceptual Framework for the International Classification for Patient Safety Version 1.1 Final Technical Report.

[20] WHO (2009c). Medicines use in primary care in developing and transitional countries. Fact Book summarizing results from studies reported between 1990 and 2006.

[21] WHO (2009d). African partnership for patient safety: working for safer health care ... together. World Health Organization, 20 Avenue Appia, 1211 Geneva 27, Switzerland

[22] WHO (n.d. b). http://www.who.int/patientsafety/research/evidence_of_unsafe/en/index.html 